\title{
The study of chain link/guide contact in relative alternative translational motion
}

\author{
Radu Papuc ${ }^{1 *}$, Cătălin Gavrilă², Mihai-Tiberiu Lateș ${ }^{3}$ \\ ${ }^{1}$ Transilvania University of Brasov, radu.papuc@unitbv.ro, Romania \\ ${ }^{2}$ Transilvania University of Brasov, cgavrila@unitbv.ro, Romania \\ ${ }^{3}$ Transilvania University of Brasov, latesmt@unitbv.ro, Romania
}

\begin{abstract}
The paper presents the study of the chain link/guide contact in relative alternative motion, by performing test and finite elements modeling. According to these, the paper presents the variation of the dynamic friction coefficient at the contact between the guide and chain links. Also, it is followed an equivalent stresses study as well as of the contact pressures variation. The conclusions are presented at the final of paper.
\end{abstract}

\section{Introduction}

The concepts evolution on friction nature creates conditions in order to better explain the notion of dynamic friction coefficient T. Fink [1].

It is seen that, so far, because of the friction phenomena complexity, an unanimously accepted theory that will explain the dependence of the friction coefficient on the intervening factors was not accepted D. H. Gordon, S. N. Kukureka [2]. But, there were experimentally determined, in different conditions, various numerical values of friction coefficients $H$. Haixia, Y. Sirong, W. Mingyu, L. Kaixin, [3].

For a more complete characterization of the dynamic friction coefficient, next there will be presented the experimental determinations for the friction coefficient between the toothed link and a guide plate by considering two testing solutions. Further, by finite elements modeling, the local contact pressures are determined.

\section{Description of testing designed models}

In order to determine the dynamic friction coefficient between the tensioning guide and toothed chain, used in an automobile transmission, there were designed and physically built two test models.

In Fig. $1-5$, there are presented the used models for determining the friction coefficient, in laboratory conditions. All models were designed through CATIA
V5 software and were machined in the workshops of Transilvania University of Brasov, Romania.

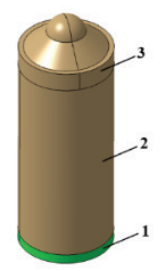

Fig. 1. Gripping - fixing device - cylinder type: 1 - guide; 2 gripping device; 3 - applying force element.

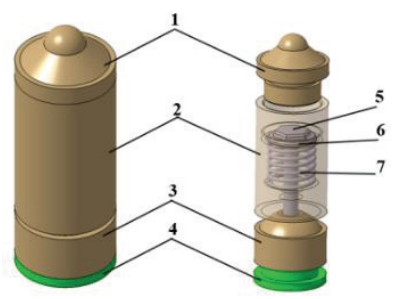

Fig. 2. Gripping - fixing device - spherical joint cylinder type: 1 - applying force element; 2 - fixing device; 3 -mobile gripping device; 4 - guide; 5 - stopping screw; 6 - washer; 7 spring.

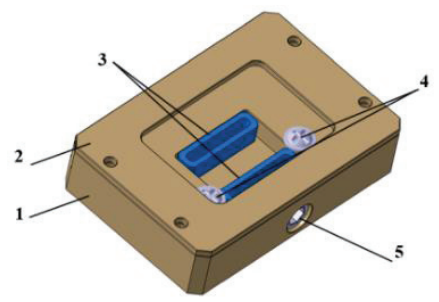

Fig. 3. Gripping - fixing device- the oil bath type: 1 - oil bath tub; 2 - bath lid; 3 - positioning and fixing screws; 4 -elastic piece; 5 -fixing screw with interior hexagonal head.

\footnotetext{
Corresponding author: radu.papuc@unitbv.ro
} 
In designing and manufacturing these devices was taken into consideration the identifying of some solutions as close as possible to the real case when the chain is in contact with the tensioning guide.

For a detailed image of the two cases presented previously, in Fig. 4 and Fig. 5 are shown, in testing position, the previous devices.

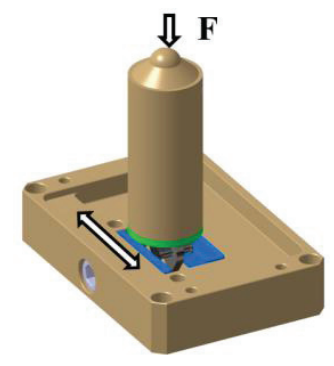

Fig. 4. The gripping - fixing devices - cylinder type

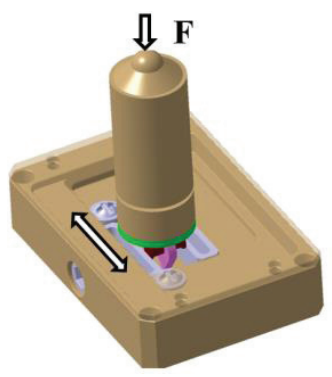

Fig. 5. The gripping - fixing devices - spherical joint cylinder type

In order to maintain the lubrication between the toothed links and the guide, it was designed a system made from two elastic elements that fixates the links into the oil bath shown in Fig. 3.

To measure the friction coefficient was used a measuring device named UMT tribometer, shown in Fig. 6 , which is connected to a computer and allows to measure and to show the friction coefficient.

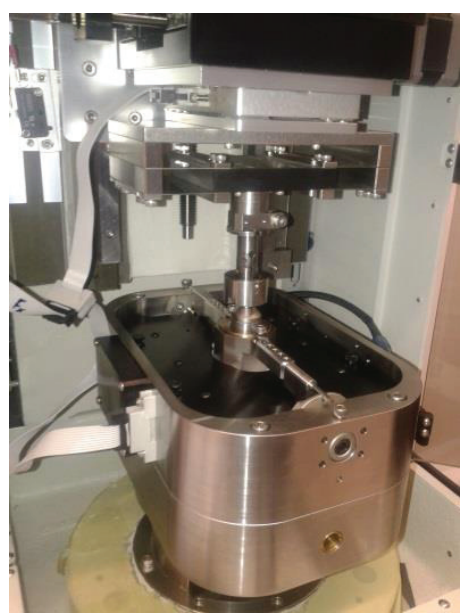

Fig. 5. The UMT tribometer

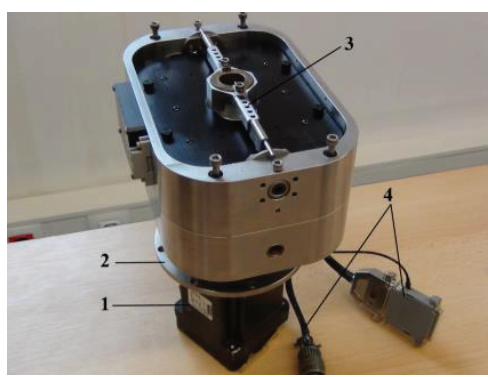

Fig. 6. The reciprocating module: 1 - command engine; 2 fixing basis; 3 - friction force reading sensor; 4 - data cables.

In order to determine the friction coefficient, the reciprocating module is attached to the tribometer. This module performs an alternative rectilinear motion while the sensor 3 is measuring the friction force.

The figures from bellow present the oil bath and the devices used to determine the relative friction coefficient, in lubricated environment, for the contact between the circular guide segments (PAx and PA66) and the toothed chain links.

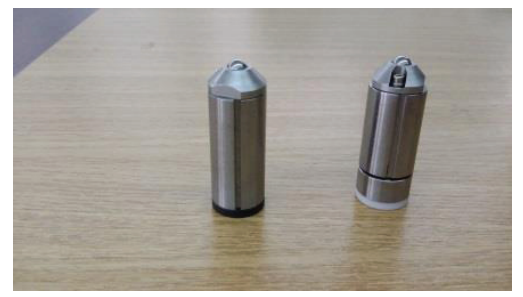

Fig. 7. The manufactured gripping - fixing devices - cylinder type and spherical joint cylinder type

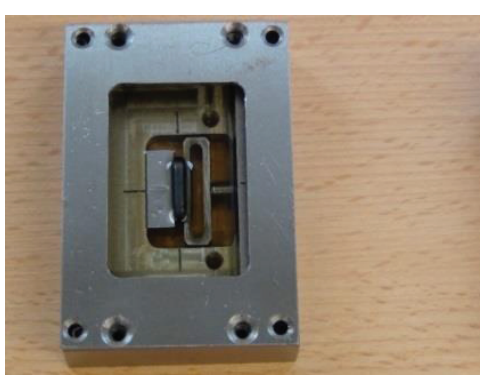

Fig. 8. The manufactured gripping - fixing device - oil bath type.

To determine the friction coefficient in lubricated environment between the toothed links and guide segment $(\mathrm{PAx})$, the following initial conditions were imposed: the normal force $\mathrm{Fn}=9(\mathrm{~N})$, which it corresponds a medium pressure pmed $=0,775(\mathrm{MPa})$; the module stroke $\mathrm{LV}=4,8(\mathrm{~mm})$; the working frequency $\mathrm{f}=1(\mathrm{~Hz})$; the test period $\mathrm{t}=2(\mathrm{~h})$; the lubricant: $15 \mathrm{~W}-40$ mineral oil.

It must be highlighted that the results regarding the obtained values for friction coefficients are presented, with some exceptions, as relative values or percentage, representing the parameters dependence for which absolute values are used; this fact was imposed by privacy clauses.

\footnotetext{
* Corresponding author: radu.papuc@unitbv.ro
} 


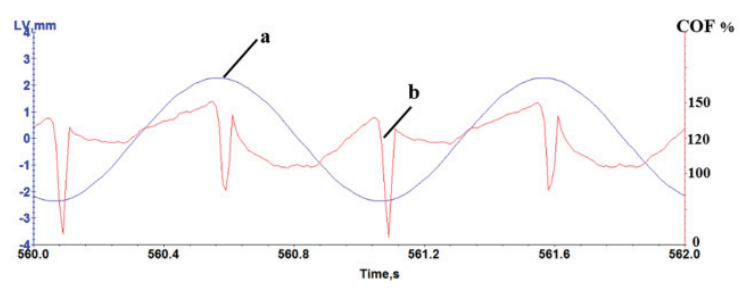

Fig. 9. The relative dynamic friction coefficient variation and the stroke for the contact between the guide segment and toothed links when using the spherical joint cylinder.

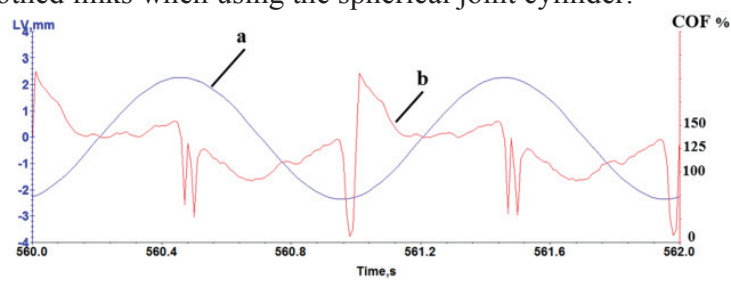

Fig. 10. The relative dynamic friction coefficient variation and the stroke for the contact between the guide segment and toothed links when using the simple cylinder.

There were performed two experimental tests, for the relative dynamic friction coefficient variations (the friction coefficient values were reported to their lowest value) between the guide and toothed links which are shown in Fig. 9 and Fig. 10. In the graphs, a is the stroke (alternative rectilinear motion) $\mathrm{LV}$, and $\mathrm{b}$ is the relative dynamic friction coefficient COF.

By analysing in comparison the test results for the cases presented in Fig. 9 and Fig. 10 it can be concluded that on the reciprocating module the relative dynamic friction coefficient can not be rigorous determined, no matter if the the guide segment having circular form is pressed on the toothed links, by having a cylindrical holder with or without a spherical joint. This conclusion is motivated on the fact that the speed corresponding to the small linear displacement made by the module is much too small and has a constant value for a short amount of time in order to obtain a conclusive representation of the relative dynamic friction coefficient values, which later will be compared with the data found in the speciality literature.

\section{The study of equivalent tensions and contact pressures}

For the numerical determination was chosen the model from figure 5 due to its complexity.

This analysis assumes the deformation state and the contact distribution between the components, guide links.

For the numerical evaluation of the studied models was used the finite element software ANSYS Workbench version .

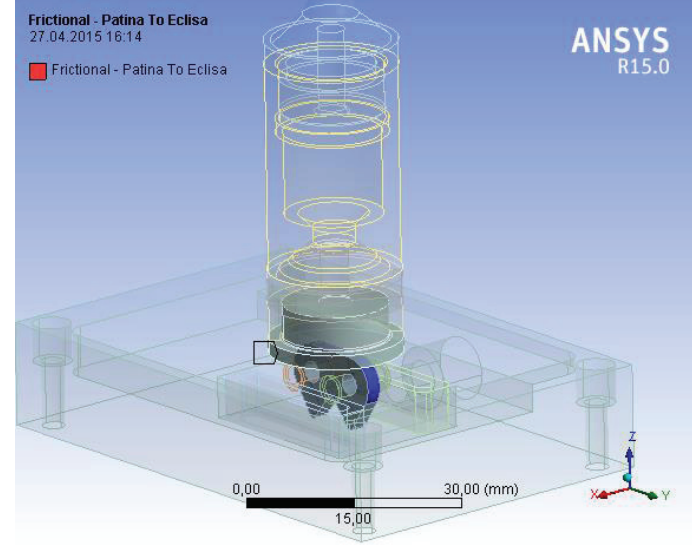

Fig. 11. Defining the contacts

In Fig. 11 is presented the defining contacts between guide and toothed links. The models were meshed with second order solids deformable finite elements with intermediary node, and for the components defined as rigide, the software used in meshing the rigide type elements J. Fish, T. A. Belychko [4].

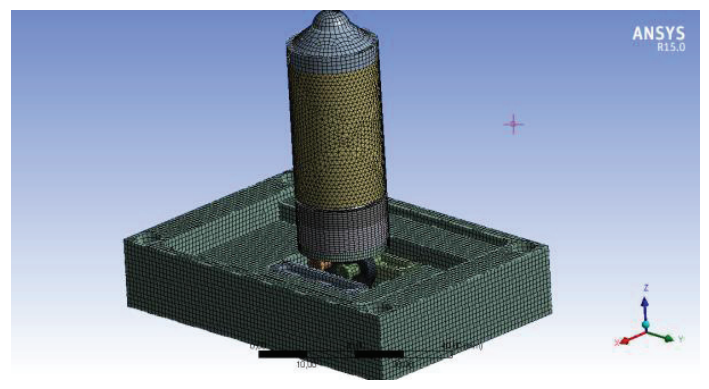

Fig. 12. Finite elements model.

The materials used in this analysis were applied to both models as follows:

1) for the guide it was used the PAx polyamide with the following properties M. Akiyama, T. Yamaguchi [5]:

a) Young module $E=2700(\mathrm{MPa})$

b) Tensile Yield Strength $\sigma=34(\mathrm{MPa})$

c) Poisson coefficient $v=0.38$

2) for other components it was used steel with the following properties A. Todi-Eftimie, R. Velicu, C. Brands, F. Schlerege, M. T. Lateș [6]:

a) Young module $E=190000(\mathrm{MPa})$

b) Tensile Yield Strength $\sigma=240(\mathrm{MPa})$

c) Poisson coefficient $v=0.3$

The model was loaded with a static force $F=9(\mathrm{~N})$, resulted from the lubricating conditions and for the contact between the toothed links and guide it was used a friction coefficient of 0.2 , determined, as well, from experimental tests.

The force is transmited from the applying point through the nonlinear contact to the guide and after to the toothed links fixed in the oil bath.

This is shown in Fig. 13. 


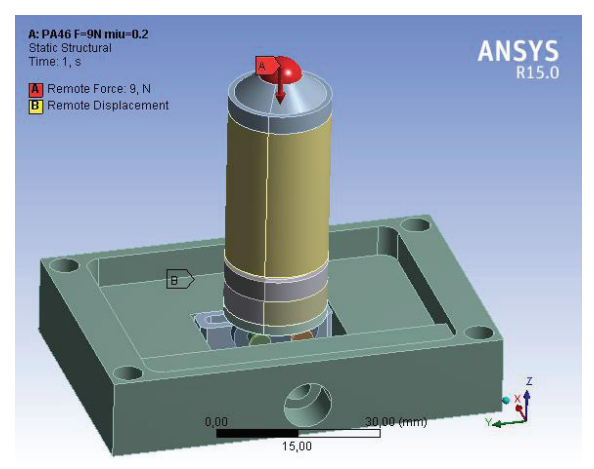

Fig. 13. Force application and freedom degrees constraints

After applying the $9(\mathrm{~N})$ force, it was obtained an uniform displacements distribution and a value of the maximum displacement which is quite small, 0.000829 (mm).

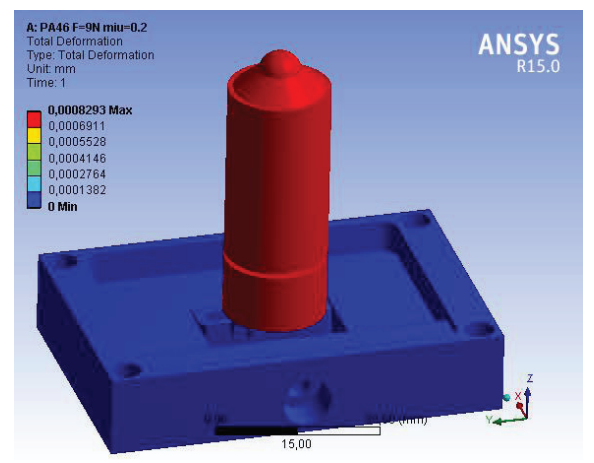

Fig. 14. Displacements distribution

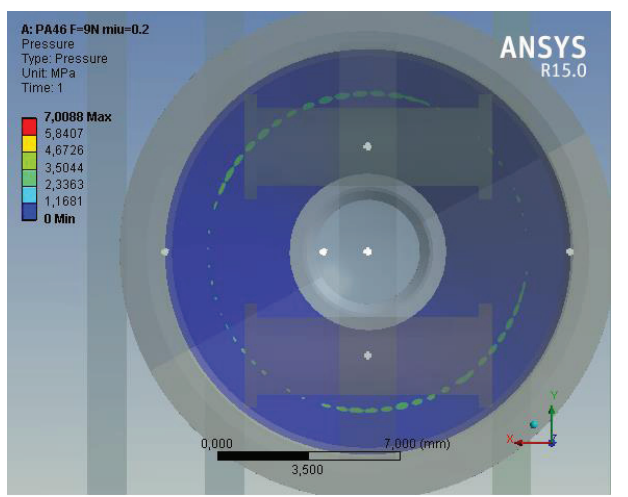

Fig. 15. The contact pressure between the mobile body and the main body.

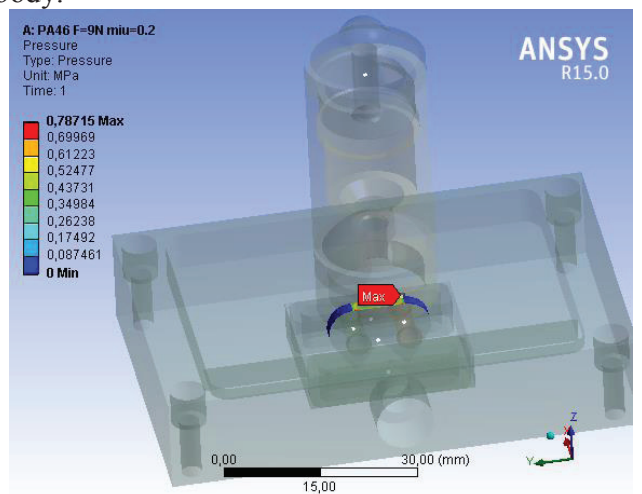

Fig. 16. The contact pressure between guide and toothed links.
This is shown in Fig. 14, where the color chart shows the precision of the model.

As it can be observed in Fig. 15, the two bodies contact pressure distribution is made on the tangent surface; the maximum value of the contact pressure in this zone is $7(\mathrm{MPa})$.

The maximum value of the contact pressure between guide and toothed links is $0.787(\mathrm{MPa})$ and is presented in figure 16.

\section{Conclusions}

The test results presented previously can be conclusive only for the start/stop situations.

This aspect is highlighted by the static friction coefficients values obtained in those situations.

By comparing these values with the speciality literature it can be concluded that in those situations it was obtained a limit friction J. Van Ruiten, R. Proost, M. Meuwissen [7].

Due to the problems identified in the reciprocating module tests, in the next stage is reccomended to perform tests on the rotary module, in order to find out the dynamic friction coefficient.

The purpose of experimental determinations for the guide - toothed links contact is to obtain a mixed friction towards fluid throughout the testing cycle and to find out the static friction coefficients which correspond to the start-stop situatuons.

Through the finite element modelling it was shown in detail the variation of the equivalent tensions and of contact pressures for the studied contact case.

\section{References}

[1] T. Fink, "Friction Reduction Potentials in Chain Drives," MTZ Worldwide Edition: Vol.72, 2011, p. 46-51.

[2] D. H. Gordon, S. N. Kukureka, „The wear and friction of polyamide 46 and polyamide $46 /$ aramid-fibre composites in sliding-rolling contact," In: Wear, 2009, p. 669-678.

[3] H. Haixia, Y. Sirong, W. Mingyu, L. Kaixin, "Tribological behavior of polyamide 66-based binary and ternary composites," In: Polymer engineering and science, 2009, p. $2454-2458$.

[4] J. Fish, T. A. Belychko, "First Course in Finite Elements," John Wiley \& Sons Publishing House, Chichester, England, 2007.

[5] M. Akiyama, T. Yamaguchi, ,Friction and wear of polyamide 66 composites filled with RB ceramics particles under dry condition," In Tribology Online No. 5 vol. 2, 2010, p. 87 - 91.

[6] A. Todi-Eftimie, R. Velicu, C. Brands, F. Schlerege, M. T. Lates, „Friction in Bearings of Parallel Axes Transmissions,” Applied Mechanics and Materials - Advanced Concepts in Mechanical Engineering I, vol. 658, 2014, ISSN 1660-9336, p. 371-376.

[7] J. Van Ruiten, R. Proost, M. Meuwissen, „How the choise of the polyamide type in timing chains tensioning systems affects the $\mathrm{CO} 2$ emission and fuel economy of internal combustion engines," In Presentation at VDI Veetiltrieb un Zylinderkopf 2012, November 28th, 2012.

\footnotetext{
${ }^{*}$ Corresponding author: radu.papuc@unitbv.ro
} 